\title{
The Value of Ultrasound Elastography in Evaluating Inflammatory Breast Lesions
}

\author{
BASMA M. ALKALAAWY, M.D.; SOHA T. HAMED, M.D.; NOHA M. ELSAYED AHMED, M.Sc. and \\ DALIA S. ELMESIDY, M.D.
}

The Department of Diagnostic and Interventional Radiology, Faculty of Medicine, Cairo University

\begin{abstract}
Background: Differentiating benign from malignant breast inflammatory conditions is crucial because they have totally different prognosis and management. Ultrasound Elastography can be used as a noninvasive tool for assessing inflammatory breast lesions. Strain Elastography can help pickup malignant inflammatory breast lesions and subsequently guide biopsy, while ruling out benign inflammatory lesions and sparing the patient unnecessary procedures.
\end{abstract}

Aim of Study: Assess the diagnostic performance of strain ultrasound elastography added to the conventional B-mode ultrasound in evaluating inflammatory breast lesions.

Patients and Methods: This study includes 33 female patients presenting with mastitis in the time period from September 2018 to October 2019. All breast lesions were assessed by Conventional B-mode ultrasound and Strain Elastography. Qualitative color-coded E-scoring and Semiquantitative SR (strain ratio) evaluation of the sonoelastographic images were performed. The results were compared to histopathologic diagnoses or follow-up for 2 weeks after antibiotic therapy, serving as reference standard.

Sensitivity, specificity, positive predictive values (PPV), negative predictive value (NPV) and total accuracy were calculated for conventional B-mode ultrasound, and Strain elastography separately and then combined together.

Statistical analysis was done using IBM@ SPSS $\odot$ Statistics version 22 (IBM@ Corp., Armonk, NY, USA). Kappa test tests were two-tailed. A $p$-value $<0.05$ was considered significant.

Results: Combined US and Elastography yielded the highest accuracy $(96.2 \%)$ and Specificity $(96 \%)$. E-score qualitative elastography yielded better accuracy (90.9\%) and Specificity $(90.3 \%)$ than Strain ratio, semiquantitative elastography.

Key Words: Inflammatory breast lesions - Ultrasound Elastography.

Correspondence to: Dr. Basma M. Alkalaawy,

The Department of Diagnostic and Interventional Radiology, Faculty of Medicine, Cairo University

\section{Introduction}

INFLAMMATORY breast lesions can be caused by a wide range of etiological factors, whether infectious, non infectious or malignancy. In some cases, clear cut clinical and imaging differences between benign and malignant mastitis can be challenging which may hinder the diagnosis and management of these patients [1].

Ultrasound Elastography offers a non-invasive tool for assessment of breast lesions through evaluating lesion elasticity/stiffness because malignant lesions tend to be more stiff (harder) than benign ones. It has two main techniques, Strain elastography (SE) that uses vibration energy either by external manual compression or intrinsic movement such as breathing and Shear wave elastography (SWE) in which the ultrasound machine itself provides the vibration energy [2].

This is a prospective study to assess the added value of Ultrasound Elastography in terms of accurate diagnosis and consequent guidance for management planning of inflammatory Breast lesions.

\section{Material and Methods}

This prospective study includes 33 female patients presenting with mastitis during the time period from September 2018 to October 2019. Their age ranged from 19 to 70 years with mean

\footnotetext{
Abbreviations:

US : Ultrasound.

SR : Strain ratio

ROI : Region of interest.

PPV : Positive predictive value.

NPV : Negative predictive value.

DLBC : Diffuse large B-cell lymphoma.

IDC : Invasive duct carcinoma.
} 
age $36.6 \pm 10$. The patients were referred from Kasr Alainy Breast outpatient clinic to the Radiology (Breast imaging) department. Patients presenting with mastitis were included whereas those presenting with pathologically proven inflammation or malignancy were excluded from the study.

All patients were examined using conventional B-mode US as well as Strain US Elastography after an informed written consent was obtained from the patientswith approval of our institutional ethical committee. Examinations, both B-mode US and elastography, were performed by two consultants of radiology, with at least 10 years of experience in breast imaging, who were blinded to each other's analysis.

Final diagnosis, our gold reference standard, was made upon follow-up for 2 weeks after antibiotic treatment or on the histopathological diagnosis of biopsy specimens. Biopsy was performed for lesions that were considered suspicious or didn't improve on empirical antibiotic treatment.

Examinations were carried out using a highend ultrasound system General Electric (GE) Logic 7 \& Logic 8, using multi-frequency linear probe operating at 6 to $15 \mathrm{MHz}$. The scanning protocol included transverse and longitudinal real-time imaging of areas of concern. A split-screen imaging mode (twin images) was used for conventional US and US elastography so as to obtain identical images optimal for accurate application for region of interest (ROI) and strain ratio (SR) measurement later on.

\section{$B$ mode US:}

Some or all of the following signs (that indicate inflammation) were confirmed:

- Echogenic, edematous fat lobules.

- Interstitial edema.

- Ill-defined collections and well defined abscess cavities.

- Retro areolar duct system dilatation.

- Thickened skin (>2mm).

- Mass lesions whether cystic or solid in nature, shape, boundary, orientation, margin, echo pattern, and posterior acoustic features, \pm calcifications.

Lesions were classified according to the American College of Radiology Breast Imaging Reporting and Data System (BI-RADS) for B-mode ultrasonography as follows: Category 2 lesions were classified as benign; category 3 as probably benign; category 4 as suspicious for malignancy; category 5 as highly suggestive of malignancy.

\section{Ultrasound elastography:}

Strain elastography examinations were carried out through qualitative color coded Elasticity soring (E-score) and the semiquantitative strain ratio (SR) measurement. Regarding E-scoring, images were obtained by placing the transducer with coupling gel on the skin and then the lesion is subjected to repeated manual compression and decompression. Color-coding is superimposed on the translucent B-mode images. According to Itoh et al., [3] images obtained in the early phase of compression were selected because these images provide the best contrast. Images were generated in a color range from red to blue. The softest part of the lesion was illustrated in red, showing the highest strain, whereas the hardest part with almost no strain was illustrated in blue. Areas of intermediate elasticity were shown in green. Elasticity scoring (E-score) was based on 5-point scoring method (Tsukuba scoring system) proposed by Itoh et al., [3], where Score $1,2, \& 3$ are considered benign and score $4 \& 5$ malignant.

- Score 1 indicated even strain for the entire lesion (i.e., the entire lesion was evenly shaded in green).

- Score 2 indicated strain in most of the lesion, with some areas of no strain (i.e., the lesion had a mosaic pattern of green and blue).

- Score 3 indicated strain at the periphery of the lesion, (i.e., the peripheral part of lesion was green, and the central part was blue).

- Score 4 indicated no strain in the whole lesion (i.e., the whole lesion was blue, but its surrounding area was not included.

- Score 5 indicated no strain in the entire lesion or in the surrounding area (i.e. the whole lesion and the surrounding breast tissue were blue).

To calculate the Strain ratio (SR), normalappearing breast region approximately at the same level of the concerned lesion was elicited as an internal reference (ROI 1) and the lesion was selected as region of interest 2 (ROI 2), to correctly determine the difference in hardness of the lesion compared with the surrounding normal tissue. The strain ratio was automatically obtained.

ROC curve and Cut off-value:

Receiver operator characteristic (ROC) curve plotting sensitivity against (1-specifity) of US BIRADS classification, E-score and Strain ratio is shown in (Fig. 1) below. Area under the curve 
was $0.9,0.92$, and 0.95 for BIRADS, strain ratio and E-score respectively. This in turn rejects the null hypothesis (area $=0.5$ ) with a significantp value of $0.05,0.04$ and 0.03 respectively. Interpretation of the ROC curve shows the following cut-off values; BIRADS (4), E-score (4) and strain ratio of (3.85).

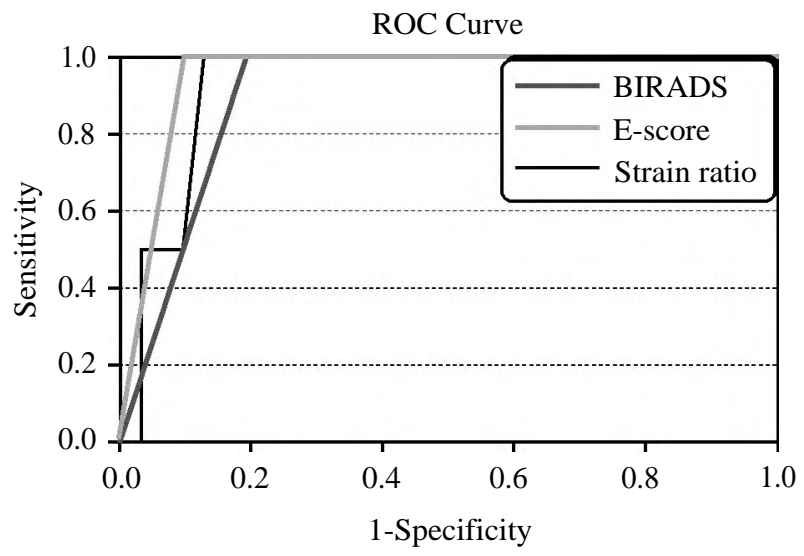

Fig. (1): ROC curve of BIRADS, E-score and Strain ratio.

\section{Results}

The patients' age ranged from 19 to 70 years with mean age $36.6 \pm 10$ (mean \pm SD). Eighteen patients $(54.5 \%)$ presented with a breast lump, while fifteen patients $(45.5 \%)$ presented with diffuse breast swelling. Patients had variable risk factors including diabetes (nine patients), lactation (eight patients), five patients (15.2\%) had previous breast surgery (CBS), and one patient (3\%) gave history of previous TB infection.

10 cases showed complete resolution after antibiotic therapy (30.3\%), whereas Tru-cut biopsy or fine needle aspiration cytology was performed for 23 cases $(69.7 \%)$, as shown in Table (1).

Table (1): Shows the final diagnosisof the patients participating in the study. In patients with breast abscess (10 cases) follow-up after 2 weeks showed complete resolution after empirical antibiotic treatment. For the rest of the cases (23 cases) histopathological diagnosis was made by means of true cut biopsy or fine needle aspiration cytology.

\begin{tabular}{lcl}
\hline Diagnosis & Number of patients $(\%)$ \\
\hline Benign: & $10 / 33$ & $(30.4 \%)$ \\
Breast abscess & $7 / 33$ & $(21.2 \%)$ \\
Granulomatous mastitis & $6 / 33$ & $(18.2 \%)$ \\
Fat necrosis & $4 / 33$ & $(12.1 \%)$ \\
Simple mastitis & $2 / 33$ & $(6.1 \%)$ \\
Infected sebaceous cyst & $1 / 33$ & $(3 \%)$ \\
Post-Irradiation changes & $1 / 33$ & $(3 \%)$ \\
TB axillary adenitis & & \\
Malignant: & $1 / 33 \quad(3 \%)$ \\
Invasive duct carcinoma & $1 / 33 \quad(3 \%)$ \\
Diffuse large B-cell lymphoma & \\
\hline
\end{tabular}

Over half of the patients $(54.5 \%)$ presented with breast lump as their main concern. These lesions proved to be benign, as shown in Table (2). On the other hand, the two patients presenting with malignancy had diffuse inflammation.

Table (2): Presents the number of patients presenting with breast mass as the dominant feature and their final diagnosis.

\begin{tabular}{lc}
\hline Diagnosis & $\begin{array}{c}\text { No. of patients presenting } \\
\text { with masses (\%) }\end{array}$ \\
\hline Breast abscess & $8 / 18(44.4 \%)$ \\
Fat necrosis & $6 / 18(33.4 \%)$ \\
Granulomatous mastitis & $2 / 18(11.1 \%)$ \\
Infected sebaceous cyst & $2 / 18(11.1 \%)$ \\
\hline
\end{tabular}

Conventional B-mode US and Semi quantitative SR Elastography:

Most of the lesions ( $81.8 \%$ ) were categorized as benign on conventional B-mode US as shown in Table (3). Most of these benign lesions were classified as BIRADS 3, whereas most of the USdiagnosed malignant lesions were classified as BIRADS 4.

Table (3): Shows BIRADS classification of breast lesions by conventional B-mode US.

\begin{tabular}{cc}
\hline BIRADS & No. of patients $(\%)$ \\
\hline Benign: & $2(6.1 \%)$ \\
II & $25(75.7 \%)$ \\
III & \\
Malignant: & $5(15.2 \%)$ \\
IV & $1(3 \%)$ \\
V & \\
\hline
\end{tabular}

Semi-quantitative SR elastography revealed wide range of SR values from 0.2 to 10 as shown in Table (4). The range for benign lesions was between 0.2 and 10 with a mean strain ratio of 2 and a standard deviation of $( \pm 2)$. On the other hand, malignant lesions showed a strain ratio of 4 for DLBCL and 7 for invasive duct carcinoma, with an average strain ratio of 5.5 and a standard deviation of $( \pm 2.1)$.

Table (4): Shows mean strain ratio according to diagnosis.

\begin{tabular}{lc}
\hline Diagnosis & Mean strain ratio (range) \\
\hline Breast abscess & $1.2(0.2-2.2)$ \\
Granulomatous mastitis & $1.6(0.4-3.7)$ \\
Fat necrosis & $4.6(0.8-10)$ \\
Simple mastitis & $1.6(0.3-3.4)$ \\
Infected sebaceous cyst & $1.2(0.8-1.7)$ \\
Post-irradiation changes & 2.7 \\
TB axillary adenitis & 0.2 \\
Invasive duct carcinoma & 7 \\
DLBCL & 3.9 \\
\hline
\end{tabular}


Both conventional B-mode US and Semi quantitative strain ratio Elastography showed similar results (using SR cutoff value of 3.85). Out of 33 patients, 27/33 lesions were diagnosed as benign, all of which turned out to be benign by Pathology $(\mathrm{NPV}=100 \%) .6$ patients were diagnosed as malignant, only 2 of them had malignant pathology (PPV=33.3\%;), one patient with IDC, and another with DLBCL. The remaining 4 patients $(66.7 \%$; false positive) had benign pathology, which proved to be fat necrosis. The calculated sensitivity of conventional US and Semi quantitative SR elastography was $100 \%$, specificity was $87 \%$, and the total accuracy was $87.8 \%$. The PPV and NPV were $33.3 \%$, and $100 \%$ respectively.

\section{Qualitative elastography:}

The distribution of elastography scores for different breast lesions isillustrated in Table (5). Lesions that scored $1,2, \& 3$ were considered benign ( $28 / 33$ cases, $84.8 \%)$, whereas lesions that scored 4 (5/33 cases, $15.2 \%)$ were considered malignant.

Table (5): Shows number of patients according to Qualitative Elastography.

\begin{tabular}{cc}
\hline E-Score & No. patients (\%) \\
\hline Benign: & $3(9.1 \%)$ \\
I & $19(57.5 \%)$ \\
II & $6(18.2 \%)$ \\
III & \\
Malignant: & $5(15.2 \%)$ \\
IV & \\
\hline
\end{tabular}

Qualitative E-score US showed slightly better results. Out of 33 patients, 28 lesions $(84.8 \%)$ had benign E-score, all of which turned out to be benign by Pathology (100\% NPV). Five patients (15.2\%) were diagnosed as malignant and had E-score of 4. Two of these 5 (40\% PPV) had malignant pathology as IDC and DLBCL. The remaining 3 patients had benign pathology ( $60 \%$; false positive), two cases of fat necrosis and one case of granulomatous mastitis. The calculated sensitivity of Escore was $100 \%$, specificity was $90.3 \%$, and the total accuracy was $90.9 \%$. The PPV and NPV were $40 \%$, and $100 \%$ respectively.

The calculated sensitivity of Combined US/ Elastography was $100 \%$, specificity was $96 \%$, and the total accuracy was $96.2 \%$. The PPV and NPV were $66.7 \%$, and $100 \%$ respectively.

Analyzing 10 patients with breast abscess, results show that the strain ratio ranged between 0.2 and 2.2 with a mean of 1.2 and standard deviation of \pm 0.6 . On the other hand, qualitative elastography results showed that $8 / 10$ patients $(80 \%)$ had an E-score of 2. One patient (10\%) had an Escore of 1 and another had an E-score of 3.

Patients with granulomatous mastitis had a SR ranging between 0.4 and 3.7 with a mean of 1.6 and standard deviation of \pm 1 .2. On performing qualitative elastography results showed that $4 / 7$ patients $(57.1 \%)$ had an E-score of 2. One patient had an E-score of 1, another had an E-score of 3 and finally one patient with an E-score of 4.

Fig. (2): 45 year old patient presenting with right breast lump. She has history of conservative breast surgery. Bmode US (A) Shows irregular lesion with heterogeneous echogenicity, ill-defined margins, showing peripheral hypervascularity (B), SR $6.2(\mathrm{C})$, and E-score 4 (D). US guided core biopsy was performed and histopathological analysis revealed fat necrosis.
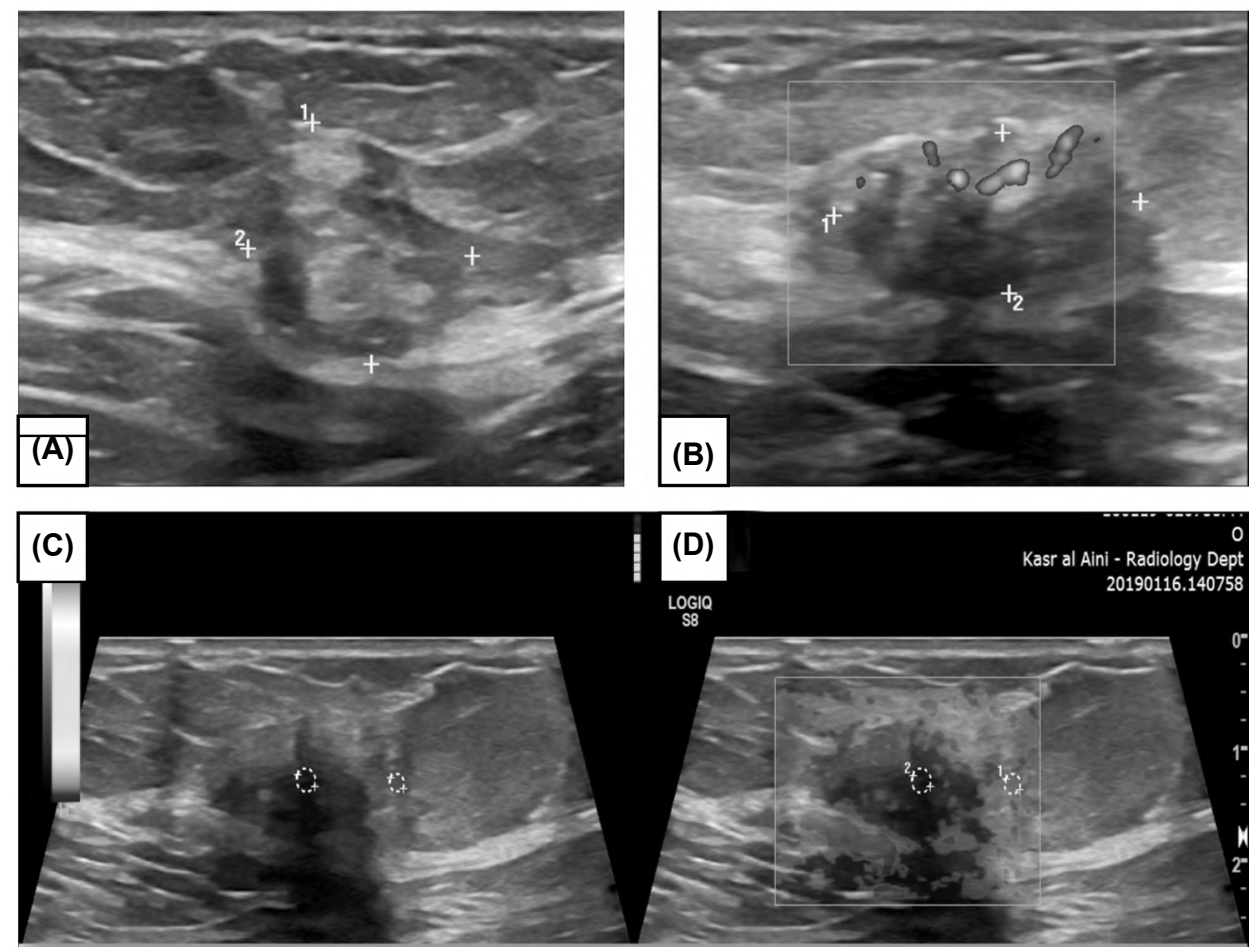
The strain ratio in patients with fat necrosis ranged between 0.8 and 10 with a mean of 4.6 and standard deviation of \pm 3.3 . Qualitative elastography results showed that $3 / 6$ patients $(50 \%)$ had an Escore of 2. One patient had an E-score of 3 and 2 patients had an E-score of 4.
On analyzing 4 patients with simple mastitis, results show that the strain ratio ranged between 0.3 and 3.4 with a mean of 1.6 and standard deviation of \pm 1.3 . Qualitative elastography results showed that $2 / 4$ patients $(50 \%)$ had an E-score of 2. One patient had an E-score of 1 and another had an E-score of 3.
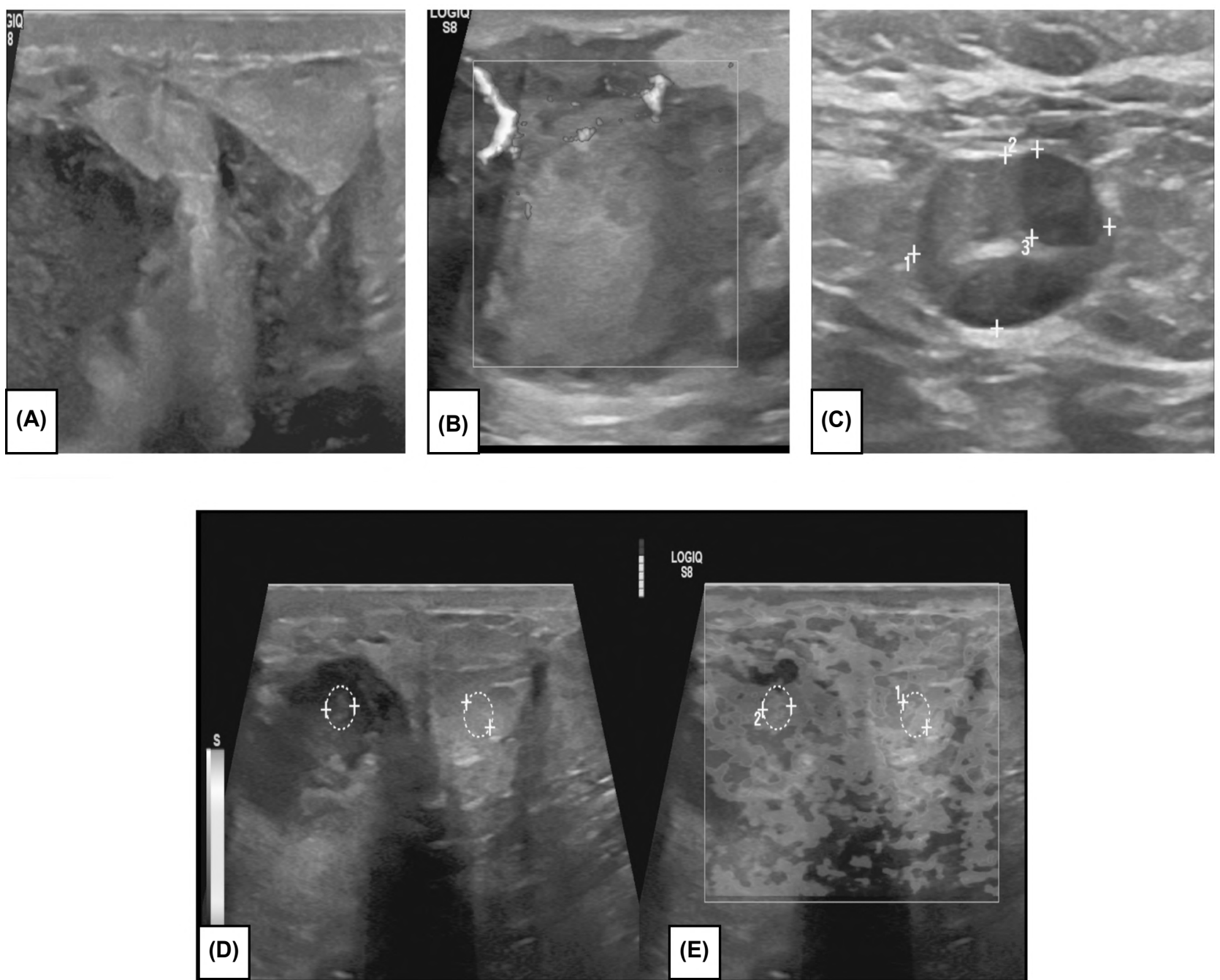

Fig. (3) 38 year old diabetic female patient presenting with right breast lump and mastalgia resistant to antibiotic treatment of one month duration. B-mode US (A) Showed large hypoechoic retroareolar lesion with echogenic fat lobules and intercommunicating ill-defined collections associated with focal skin thickening measuring $4.6 \mathrm{~mm}$ and increased vascularity on color Doppler application (B). Lesion was classified as BIRADS IV. Ipsilateral axillary lymph nodes (C) were enlarged with diffuse cortical thickening reaching $6 \mathrm{~mm}$ with effaced fatty hilum. SR was 1.5 (D), and E-score was 2 (E). Ultrasound guided core biopsy revealed granulomatous mastitis. 

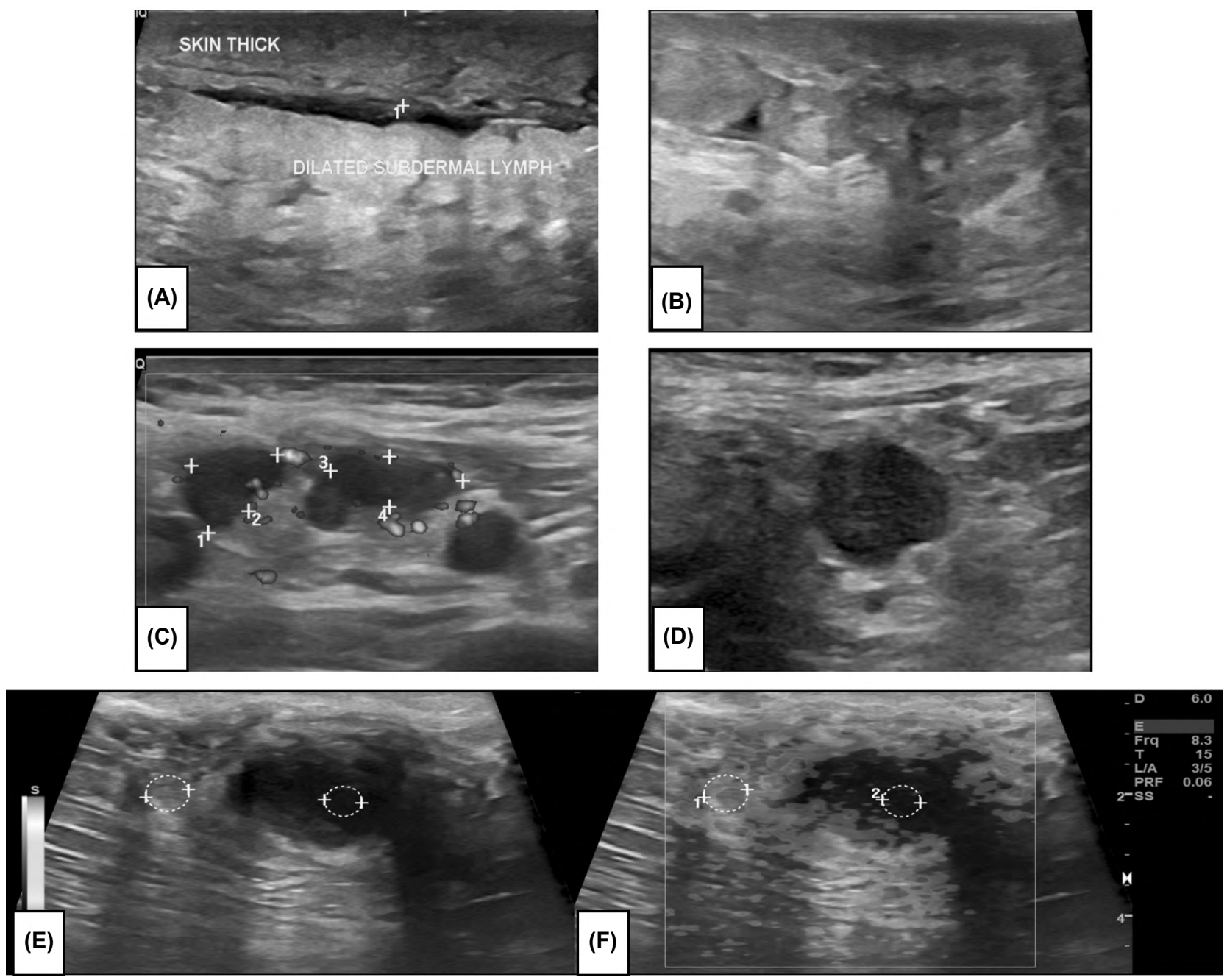

Fig. (4): 35 years old female, presented with left breast swelling and mastalgia of 2 months duration with no response to medical treatment. B-mode breast US (A) \& (B) Shows marked diffuse inflammatory changes in form of echogenic fat lobules, interstitial edema, marked diffuse thickening of the overlying skin reaching $11.6 \mathrm{~mm}$ and hypervascularity on color Doppler application (BIRADS IV). (C) \& (D) reveal pathologically enlarged axillary lymph nodes. SR is 7 (E) E-score is 4 (F). US guided core needle biopsy was done and yielded invasive duct carcinoma grade II.

\section{Discussion}

Differentiating benign from malignant mastitis can present a clinical and a radiological diagnostic challenge. Both have totally different approaches and prognosis, which makes differentiation crucial. Empirical antibiotic medical treatment and watchful waiting used in benign mastitis can negatively impact inflammatory carcinoma patients $[4,5]$.

To our knowledge, few published studies were exclusively dedicated for the discussion of different breast inflammatory lesions by Strain Elastography. In the present study we evaluated the diagnostic performance of Strain elastography in different inflammatory breast conditions and its ability to pick up the malignant lesions and guide biopsy. We used the qualitative color-coded E-scoring and semi quantitative SR methods in addition to conventional B-mode ultrasound.

Our study included 7 cases of granulomatous mastitis. The strain ratio ranged from 0.4-3.7, with a mean of 1.6 $\pm 1.2(\mathrm{SD})$. All cases of granulomatous mastitis had Elasticity score of 1 to 3 and only one patient with score 4 . These results are consistent with two studies by Yag c1 et al., [6] and DururKarakaya et al., [7] investigating strain elastography in idiopathic granulomatous patients. They concluded SR of granulomatous mastitis to be $1.5 \pm$ 0.8 (SD) (range: 0.2-4) [6] and 1.10 \pm 0.79 (range 0.29-4.00) [7] and Elasticity scores between 1 and 3 [7].

Sousaris and Barr [8] conducted a study investigating the value of Strain Elastography in the 
differentiation between mastitis with abscess formation and malignancy. Their study showed abscess cavities to have a soft center and stiff rim which is a rare occurrence in malignant lesions that have central areas of necrosis. In their work, the SR of the central soft areas of these abscess cavities ranged from 1.2 to 2.0 (mean, 1.4). This is comparable to our results that showed abscess cavities to have SR ranging between 0.2 and 2.2 with a mean of 1.2.

Fat necrosis tends to be hard at palpation with false positive results on Elastography owing to stiffness of the lesion [9]. Our results showed fat necrosis had strain ratio ranging between 0.8 and 10 with a mean of 4.6 and standard deviation of \pm 3 .3. This high strain ratio is due to the fact that fat necrosis patients in our study presented at early stages of inflammation when the lesion is still hard before the eventual liquefaction into oil cysts. However, qualitative elastography results showed that 4 patients had benign elasticity score of 2 and 3 and only 2 patients had a malignant score of 4 . Raza et al., [10] had similar results where 2 out of 3 cases of fat necrosis had a benign elasticity score of 2 and one case had elasticity score of 4.

Our study included only 2 cases with inflammatory breast cancer (6\% of cases), which is justified in part by the relative rarity of the disease itself. It represents 2-3\% of breast cancers in the USA and 6-10\% in North Africa [11]. Even though, in the current study Elastography was successfully able to highlight both cases as malignant (100\% sensitivity).

As far as our results showed, conventional Bmode US and Semi quantitative SRElastographyperformed similarly yielding sensitivity of $100 \%$, specificity of $87 \%$, and total accuracy of $87.8 \%$. The PPV and NPV were $33.3 \%$, and $100 \%$ respectively. Meanwhile, we observed better results with Qualitative E-score with 100\% sensitivity, 90.3\%, specificity and $90.9 \%$ total accuracy. The PPV and NPV were $40 \%$, and $100 \%$ respectively.

Different SR cutoff values were used in different studies influenced by the US equipment used from different manufacturers as well as the study population itself [12]. On implementing cut off values of 3.85 for semiquantitative SR, and score of 3 for qualitative E-score, above which we considered the lesion malignant, we achieved results comparable to many studies [13-19] evaluating strain elastography for differentiating benign from malignant breast lesions.
Our results are in concordance with a variety of studies using SR cutoff values close to ours. Yerli et al., [14] used SR cut off value of 3.52 and reported $80 \%$ sensitivity, 93\% specificity, 93\% NPV, while Mutala et al., [13] used SR cutoff value 4.2 with reported $93 \%$ sensitivity, $96 \%$ specificity and $96 \%$ NPV. Also Houelleu Demay et al., [19] achieved $99 \%$ specificity when using SR value of 3.05.

In our study, on combining B-mode US and Elastography the sensitivity was not affected remaining at $100 \%$. On the other hand specificity improved to $96 \%$, as did the PPV and NPV to $66.7 \%$ and $100 \%$ respectively. Similar conclusion was suggested by Zhao et al., [16] where adding SR to routine US examination improved the specificity of breast US lesion assessment without a loss of sensitivity. Bojanic et al., [20] also concluded that combined US and SE yielded better results than US alone.

We concluded that Color-coded Qualitative Escore had slightly higher specificity $(90.9 \%)$ than Semi quantitative SR Elastography (87\%). Similar results were obtained by Barr et al., [21] (87\% versus $81 \%$ ), Bojanic et al., [20] (93\% versus 87.6\%), and Menezes et al., [22] (82.6\% versus 74.6) who concluded E-score to be more sensitive than SR in differentiating benign from malignant lesions. However, Mutala et al., [13] reported no significant difference between the two methods. Regarding results of our work, further larger scale studies for inflammatory breast diseases are required to compare the diagnostic performance of both examinations.

The current study has its limitations. The first and major limitation being the small size of the study population with poor representation of malignant pathology. In addition the lack of comparative studies committed to exclusive assessment of US Elastography in different inflammatory breast lesions. Last but not least the operator dependence encountered on performing any nonautomated US examination.

Future larger scale studies should be carried out to assess the added benefit of US Elastography in inflammatory breast lesions. Furthermore, determining a standard SR cutoff value to rule out malignant inflammation.

\section{Conclusion:}

Strain Elastography using color-coded Elasticity score and SR is a valuable noninvasive addition to routine breast US in evaluating different inflam- 
matory breast lesions. It has the potential to mitigate unnecessary biopsies in patients with benign inflammation and affirm the decision for biopsy in suspicious inflammatory lesions.

\section{References}

1- LEONG P.W., CHOTAI N.C. and KULKARNI S.: Imaging Features of Inflammatory Breast Disorders: A Pictorial Essay. Korean J. Radiol., 19 (1): 5, 2018.

2- SIGRIST R.M.S., LIAU J., KAFFAS A. EL, et al.: Ultrasound Elastography: Review of Techniques and Clinical Applications. Theranostics, 7 (5): 1303-29, 2017.

3- ITOH A., UENO E., TOHNO E., et al.: Breast disease: Clinical application of US elastography for diagnosis. Radiology, 239 (2): 341-50, 2006.

4- KAMAL R.M., HAMED S.T. and SALEM D.S.: Classification of Inflammatory Breast Disorders and Step by Step Diagnosis. Breast J., 15 (4): 367-80, 2009.

5- LEPORI D.: Inflammatory breast disease: The radiologist's role. Diagn. Interv. Imaging, 96 (10): 1045-64, 2015.

6- YAĞ Cı B., ERDEM TOSLAK I., ÇEKIÇ B., et al.: Differentiation between idiopathic granulomatous mastitis and malignant breast lesions using strain ratio on ultrasonic elastography. Diagn. Interv. Imaging, 98 (10): 685-91, 2017.

7- DURUR-KARAKAYA A., DURUR-SUBASI I., AKCAY M.N., et al.: Sonoelastography findings for idiopathic granulomatous mastitis. Jpn. J. Radiol., 33 (1): 33-8, 2015.

8- SOUSARIS N. and BARR R.G.: Sonographic Elastography of Mastitis. J. Ultrasound Med., 35 (8): 1791-7, 2016.

9- RICCI P, MAGGINI E., MANCUSO E., et al.: Application of breast elastography: State of the art. Eur. J. Radiol., 83 (3): 429-37, 2014.

10- RAZA S., ODULATE A., ONG E.M.W., et al.: Using Real-time Tissue Elastography for Breast Lesion Evaluation. J. Ultrasound Med., 29 (4): 551-63, 2010.

11-MEJRI N., BENNA H. EL, M'GHIRBI F., et al.: Biological features of inflammatory breast cancer in North Africa: burden and research priorities. Breast Cancer Manag., 7 (2): BMT11, 2018.
12- SADIGH G., CARLOS R.C., NEAL C.H., et al.: Accuracy of quantitative ultrasound elastography for differentiation of malignant and benign breast abnormalities: A metaanalysis. Breast Cancer Res. Treat., 134 (3): 923-31, 2012.

13-MUTALA T.M., NDAIGA P. and AYWAK A.: Comparison of qualitative and semiquantitative strain elastography in breast lesions for diagnostic accuracy. Cancer Imaging, 16 (1): 12, 2016.

14- YERLI H., YILMAZ T., KASKATI T., et al.: Qualitative and Semiquantitative Evaluations of Solid Breast Lesions by Sonoelastography. J. Ultrasound Med., 30 (2): 17986, 2011.

15- KIM H.J., KIM S.M., KIM B., et al.: Comparison of strain and shear wave elastography for qualitative and quantitative assessment of breast masses in the same population. Sci. Rep., 8 (1): 6197, 2018.

16-ZHAO X., YAO J., ZHOU X.C., et al.: Strain Elastography: A Valuable Additional Method to Strain Elastografie: Eine wertvolle Zusatzmethode für, 2013: 526-34, 2018.

17- PARAJULY S.S., LAN P.Y., YAN L., et al.: Breast elastography: A hospital-based preliminary study in China. Asian Pacific J. Cancer Prev., 11 (3): 809-14, 2010.

18- MANSOUR S.M. and OMAR O.S.: Elastography ultrasound and questionable breast lesions: Does it count? Eur. J. Radiol., 81 (11): 3234-44, 2012.

19- HOUELLEU DEMAY M-L., MONGHAL C., BERTRAND P., et al.: An assessment of the performance of elastography for the investigation of BI-RADS 4 and BIRADS 5 breast lesions: Correlations with pathological anatomy findings. Diagn. Interv. Imaging, 93 (10): 757 66, 2012.

20- BOJANIC K., KATAVIC N., SMOLIC M., et al.: Implementation of Elastography Score and Strain Ratio in Combination with B-Mode Ultrasound Avoids Unnecessary Biopsies of Breast Lesions. Ultrasound Med. Biol., 43 (4): 804-16, 2017.

21- BARR R.G., De SILVESTRI A., SCOTTI V., et al.: Diagnostic Performance and Accuracy of the 3 Interpreting Methods of Breast Strain Elastography: A Systematic Review and Meta analysis. J. Ultrasound Med., 38 (6): 1397-404.

22- MENEZES R.: Correlation of Strain Elastography with Conventional Sonography and FNAC/Biopsy. J. Clin. Diagnostic Res., 10 (7): TC05-TC10, 2016. 


\section{دورقياس المرونة بالموجات فوق الصوتية

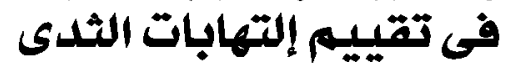

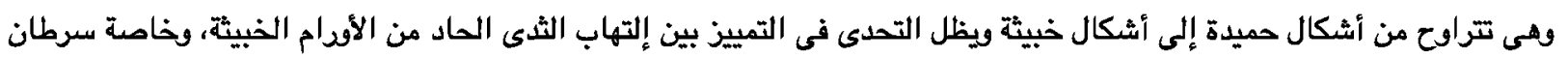

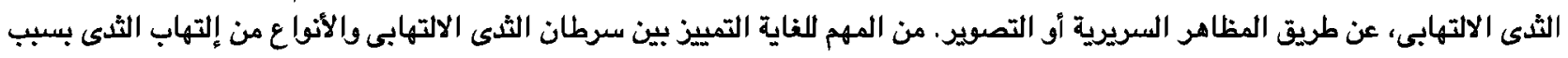
وجود اختلافات كبيرة في تشخيصنه وعلاجه.

تحتاج الموجات فوق الصوتية إلى متخصص أشعة إلى جانب معدات جيدة لتجنب التفسير الخاطئ للأفات وتخفيض عدد النقات النتائج الإيجابية

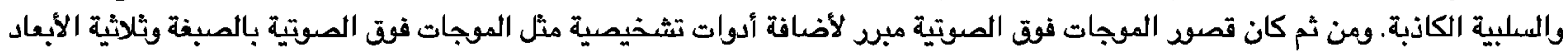
وقياس المرونة.

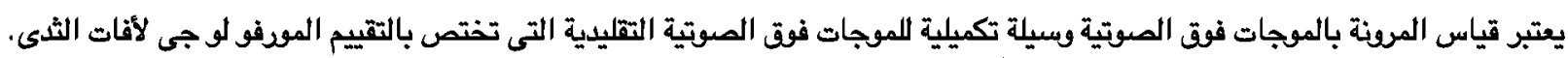

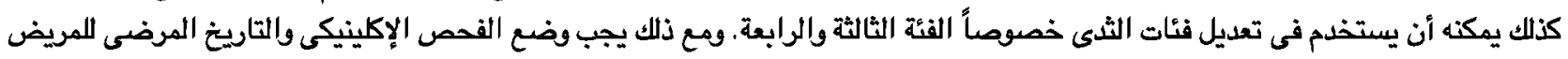

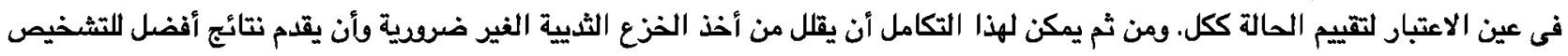

بتخفيض معدل النتائج الإيجابية الكاذبة.

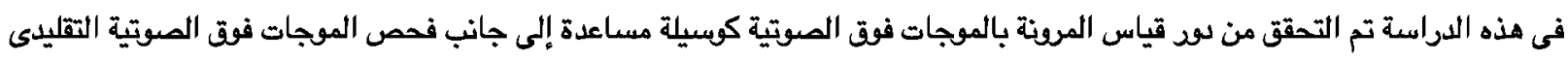

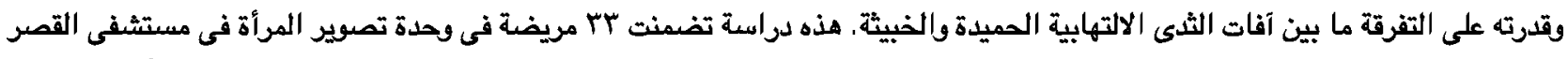

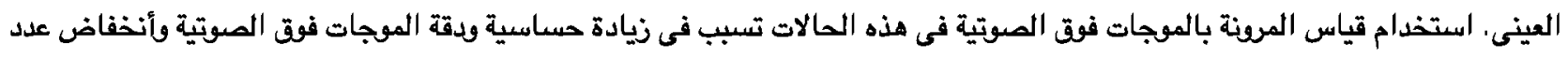

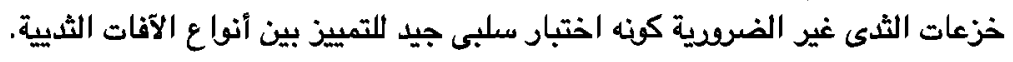

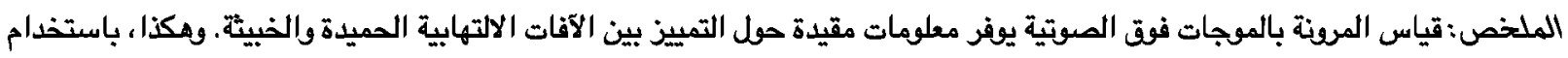

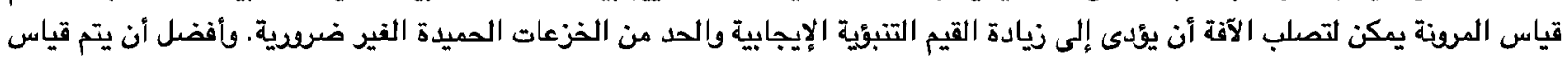
المرونة بالموجات فوق الصوتية بنوعيه الكيفى والكمى حيث أن الكيفى ألكئ أكثر دقة من الكمى. 